\title{
The Value of Collaboration in Improving Knowledge on Rare Diseases
}

Can. J. Neurol. Sci. 2011; 38: 387

The paper by Mah et al published in this edition of the Canadian Journal of the Neurological Sciences is an excellent illustration of how collaborative efforts across a clinical network are crucial in understanding rare diseases and improving standards of diagnosis and ultimately care ${ }^{1}$. The paper presents the findings of the Canadian Paediatric Neurology Group which "was founded in 2005 as a collaborative effort to promote research and to enhance the clinical care of children with neuromuscular disease". This multidisciplinary group has representation across Canada. The work described here specifically addressed the issue of diagnosis in Duchenne muscular dystrophy over a ten-year-period during which diagnostic standards have changed significantly due to advances in molecular genetic technologies.

Duchenne muscular dystrophy is one of the more common inherited diseases of childhood but nonetheless is a rare disease, with onset typically before the age of four years followed by a rapidly progressive loss of muscle strength and function and reduction in life expectancy due to respiratory and cardiac complications. An improved understanding of the molecular basis of the disease has not only led to an improved set of diagnostic techniques for confirming the diagnosis, but also has led to specific studies towards therapies, some of which are in or about to enter clinical trials ${ }^{2,3}$. In this context, the impetus to a precise diagnosis, which was initially all around issues of facilitating genetic counseling and potential prevention of secondary cases has expanded to include the delineation of a precise diagnosis that might allow the application of specific gene modification based therapies.

The prevalence of Duchenne muscular dystrophy in Canada over this period indicated a gradual increase despite the provision of genetic counseling, suggesting perhaps an improved awareness of the diagnosis. The Canadian Paediatric Neurology Group was able to show a shift towards better precision of diagnosis over the period studied, though the application of the more sophisticated tests showed significant regional variation and over Canada as a whole $26 \%$ of patients were still diagnosed at the end of the period using an outdated technique which does not in all cases allow for a comprehensive diagnosis to be reached. These figures are similar to a recent study in the $\mathrm{US}^{4}$, and indicate that there is still an important need for equity of access to the currently recommended diagnostic techniques ${ }^{5}$ as well as a continued need for education as to why their application is important for care of the patient and their family.

The types of mutations reported in the Canadian study are comparable to other studies, confirming the global impact of this disease and the distinct mutation types which may be amenable to different novel therapeutic options. Registry efforts, such as those facilitated by the paediatric network in Canada are essential to issues such as trial feasibility, patient identification and recruitment and a commitment to the highest quality genetic testing has to underpin these efforts. These principles are enshrined in the global TREAT-NMD registries (http:// www.treat-nmd.eu/resources/patient-registries/overview) to which the Canadian registry is an active contributor.

Ensuring that patients with rare diseases get access to the best quality diagnosis and care presents challenges that are best addressed by networking and sharing of resources. It is good to see this concept bear fruit in the paediatric neuromuscular community in Canada.

\section{Kate Bushby \\ Newcastle-upon-Tyne, United Kingdom}

\section{REFERENCES}

1. Mah JK, Selby K, Campbell C, et al. A population-based study of dystrophin mutations in Canada. Can J Neurol Sci. 2011;38(3): 465-74.

2. Bushby K, Finkel R, Birnkrant DJ, et al. Diagnosis and management of Duchenne Muscular Dystrophy, part 1: diagnosis and pharmacological and psychosocial management. Lancet Neurol. 2010;9(1):77-93.

3. Bushby K, Lochmuller H, Lynn S, Straub V. Interventions for muscular dystrophy: molecular medicines entering the clinic. Lancet. 2009;374(9704):1849-56.

4. Cunnif C, Andrews J, Meaney FJ, et al. Mutation analysis in a population based cohort of boys with Duchenne or Becker muscular dystrophy. J Child Neurol. 2009;24(4):425-30.

5. Abbs S, Tuffery-Giraud S, Bakker E, Ferlini A, Sejersen T, Mueller CR. Best practice guidelines on molecular testing in Duchenne and Becker muscular dystrophies. Neuromuscul Disord. 2010 Jun;20(6):422-7. 